\title{
Theoretical Characterization of End-on and Side-on Peroxide Coordination in Ligated $\mathrm{Cu}_{2} \mathrm{O}_{2}$ Models
}

Christopher J. Cramer, ${ }^{a}$ Armagan Kinal, ${ }^{b}$ Marta Włoch, ${ }^{b}$ Piotr Piecuch, ${ }^{b, c}$ Laura Gagliardi $^{d}$

a Department of Chemistry and Supercomputer Institute, University of Minnesota, 207 Pleasant St. SE, Minneapolis, MN 55455, USA

${ }^{b}$ Department of Chemistry, Michigan State University, East Lansing, MI 48824, USA

${ }^{c}$ Department of Physics and Astronomy, Michigan State University, East Lansing, MI 48824, USA

d Department of Physical Chemistry, Sciences II University of Geneva, 30 Quai Ernest Ansermet, CH-1211 Geneva 4, Switzerland

$$
\text { April 19, } 2007
$$

(correction to original version dated July 5, 2006; correction is new 100\% geometries for isomers $\mathbf{0}$ and $\mathbf{2}$ on pp. S-2 and S-4, respectively)

\section{Contents}

Cartesian Coordinates ( $0 \%$ and $100 \%)$

Singlet and Broken-symmetry Absolute $\left(E_{\mathrm{h}}\right)$ and Relative $\left(\mathrm{kcal} \mathrm{mol}^{-1}\right)$

Triplet Electronic Energies $\left(E_{\mathrm{h}}\right)$ and $<S^{2}>$ Values (for Computing Sum-bs Energies) S-18 Software 


\section{Cartesian Coordinates ( $0 \%$ and $100 \%)$}

Note that all other structures may be determined using eq. 2 of the manuscript

\begin{tabular}{|c|c|c|c|c|c|}
\hline \multicolumn{6}{|c|}{$\mathrm{Cu}_{2} \mathrm{O}_{2}{ }^{2+}(\mathbf{0})$} \\
\hline \multicolumn{6}{|l|}{$0 \%$} \\
\hline Center & Atomic & Atomic & Coor & inates (Ang & roms ) \\
\hline Number & Number & Type & $\mathrm{x}$ & $\mathrm{Y}$ & $\mathrm{z}$ \\
\hline 1 & 29 & 0 & -2.380000 & -0.410000 & 0.000000 \\
\hline 2 & 29 & 0 & 2.380000 & 0.410000 & 0.000000 \\
\hline 3 & 8 & 0 & -0.530000 & 0.410000 & 0.000000 \\
\hline 4 & 8 & 0 & 0.530000 & -0.410000 & 0.000000 \\
\hline
\end{tabular}

$100 \%$

\begin{tabular}{|c|c|c|c|c|c|}
\hline \multirow{2}{*}{$\begin{array}{l}\text { Center } \\
\text { Number }\end{array}$} & \multirow{2}{*}{$\begin{array}{l}\text { Atomic } \\
\text { Number }\end{array}$} & \multirow{2}{*}{$\begin{array}{c}\text { Atomic } \\
\text { Type }\end{array}$} & \multicolumn{3}{|c|}{ Coordinates (Angstroms) } \\
\hline & & & $\mathrm{x}$ & $\mathrm{Y}$ & $\mathrm{z}$ \\
\hline-- & -- & & --- & -- & ---ー---- \\
\hline 1 & 29 & 0 & -1.800000 & 0.000000 & 0.000000 \\
\hline 2 & 29 & 0 & 1.800000 & 0.000000 & 0.000000 \\
\hline 3 & 8 & 0 & 0.000000 & 0.700000 & 0.000000 \\
\hline 4 & 8 & 0 & 0.000000 & -0.700000 & 0.000000 \\
\hline
\end{tabular}

$\left\{\left(\mathrm{NH}_{3}\right) \mathrm{Cu}\right\}_{2} \mathrm{O}_{2}{ }^{2+}(\mathbf{1})$

$0 \%$

\begin{tabular}{|c|c|c|c|c|c|}
\hline \multirow{2}{*}{$\begin{array}{l}\text { Center } \\
\text { Number }\end{array}$} & \multirow{2}{*}{$\begin{array}{l}\text { Atomic } \\
\text { Number }\end{array}$} & \multirow{2}{*}{$\begin{array}{c}\text { Atomic } \\
\text { Type }\end{array}$} & \multicolumn{3}{|c|}{ Coordinates (Angstroms) } \\
\hline & & & $\mathrm{x}$ & $\mathrm{Y}$ & $\mathrm{z}$ \\
\hline- & --- & -- & ---- & -----ー- & -------- \\
\hline 1 & 29 & 0 & 0.593891 & 2.340449 & 0.000000 \\
\hline 2 & 29 & 0 & -0.593891 & -2.340449 & 0.000000 \\
\hline 3 & 8 & 0 & 0.593891 & 0.314449 & 0.000000 \\
\hline 4 & 8 & 0 & -0.593891 & -0.314449 & 0.000000 \\
\hline 5 & 7 & 0 & 0.593891 & 4.440449 & 0.000000 \\
\hline 6 & 7 & 0 & -0.593891 & -4.440449 & 0.000000 \\
\hline 7 & 1 & 0 & -0.345802 & 4.782469 & 0.000000 \\
\hline 8 & 1 & 0 & 0.345802 & -4.782469 & 0.000000 \\
\hline 9 & 1 & 0 & 1.063737 & 4.782469 & 0.813798 \\
\hline 10 & 1 & 0 & -1.063737 & -4.782469 & -0.813798 \\
\hline 11 & 1 & 0 & 1.063737 & 4.782469 & -0.813798 \\
\hline 12 & 1 & 0 & -1.063737 & -4.782469 & 0.813798 \\
\hline
\end{tabular}


$100 \%$

\begin{tabular}{|c|c|c|c|c|c|}
\hline \multirow{2}{*}{$\begin{array}{l}\text { Center } \\
\text { Number }\end{array}$} & \multirow{2}{*}{$\begin{array}{l}\text { Atomic } \\
\text { Number }\end{array}$} & \multirow{2}{*}{$\begin{array}{l}\text { Atomic } \\
\text { Type }\end{array}$} & \multicolumn{3}{|c|}{ Coordinates (Angstroms) } \\
\hline & & & $\mathrm{x}$ & $\mathrm{Y}$ & $\mathrm{z}$ \\
\hline 1 & 29 & 0 & 0.000000 & 1.800000 & 0.000000 \\
\hline 2 & 29 & 0 & 0.000000 & -1.800000 & 0.000000 \\
\hline 3 & 8 & 0 & 0.000000 & 0.000000 & 0.700000 \\
\hline 4 & 8 & 0 & 0.000000 & 0.000000 & -0.700000 \\
\hline 5 & 7 & 0 & 0.000000 & 3.800000 & 0.000000 \\
\hline 6 & 7 & 0 & 0.000000 & -3.800000 & 0.000000 \\
\hline 7 & 1 & 0 & -0.939693 & 4.142020 & 0.000000 \\
\hline 8 & 1 & 0 & 0.939693 & -4.142020 & 0.000000 \\
\hline 9 & 1 & 0 & 0.469846 & 4.142020 & 0.813798 \\
\hline 10 & 1 & 0 & -0.469846 & -4.142020 & -0.813798 \\
\hline 11 & 1 & 0 & 0.469846 & 4.142020 & -0.813798 \\
\hline 12 & 1 & 0 & -0.469846 & -4.142020 & 0.813798 \\
\hline
\end{tabular}

$\left\{\left(\mathrm{NH}_{3}\right)_{2} \mathrm{Cu}\right\}_{2} \mathrm{O}_{2}{ }^{2+}(\mathbf{2})$

$0 \%$

\begin{tabular}{|c|c|c|c|c|c|}
\hline \multirow{2}{*}{$\begin{array}{l}\text { Center } \\
\text { Number }\end{array}$} & \multirow{2}{*}{$\begin{array}{l}\text { Atomic } \\
\text { Number }\end{array}$} & \multirow{2}{*}{$\begin{array}{l}\text { Atomic } \\
\text { Type }\end{array}$} & \multicolumn{3}{|c|}{ Coordinates (Angstroms) } \\
\hline & & & $\mathrm{x}$ & $\mathrm{Y}$ & $\mathrm{z}$ \\
\hline$=-$ & --- & & ---- & -------- & ------ \\
\hline 1 & 29 & 0 & 1.451897 & 2.203097 & 0.093215 \\
\hline 2 & 29 & 0 & -1.451897 & -2.203097 & -0.093215 \\
\hline 3 & 8 & 0 & 0.562652 & 0.192151 & -0.257954 \\
\hline 4 & 8 & 0 & -0.562652 & -0.192151 & 0.257954 \\
\hline 5 & 7 & 0 & 3.015496 & 1.885742 & -1.059336 \\
\hline 6 & 7 & 0 & 0.191645 & 3.085434 & 1.315301 \\
\hline 7 & 7 & 0 & -3.015496 & -1.885742 & 1.059336 \\
\hline 8 & 7 & 0 & -0.191645 & -3.085434 & -1.315301 \\
\hline 9 & 1 & 0 & 3.591514 & 1.090828 & -0.742332 \\
\hline 10 & 1 & 0 & 0.076925 & 2.580102 & 2.207074 \\
\hline 11 & 1 & 0 & -3.591514 & -1.090828 & 0.742332 \\
\hline 12 & 1 & 0 & 0.750033 & -3.197954 & -0.909807 \\
\hline 13 & 1 & 0 & 3.641906 & 2.706025 & -1.078320 \\
\hline 14 & 1 & 0 & -0.750033 & 3.197954 & 0.909807 \\
\hline 15 & 1 & 0 & -2.758967 & -1.696618 & 2.040454 \\
\hline 16 & 1 & 0 & -0.076925 & -2.580102 & -2.207074 \\
\hline 17 & 1 & 0 & 2.758967 & 1.696618 & -2.040454 \\
\hline 18 & 1 & 0 & 0.514919 & 4.034607 & 1.561292 \\
\hline 19 & 1 & 0 & -3.641906 & -2.706025 & 1.078320 \\
\hline 20 & 1 & 0 & -0.514919 & -4.034607 & -1.561292 \\
\hline
\end{tabular}


$100 \%$

\begin{tabular}{|c|c|c|c|c|c|}
\hline \multirow{2}{*}{$\begin{array}{l}\text { Center } \\
\text { Number }\end{array}$} & \multirow{2}{*}{$\begin{array}{l}\text { Atomic } \\
\text { Number }\end{array}$} & \multirow{2}{*}{$\begin{array}{l}\text { Atomic } \\
\text { Type }\end{array}$} & \multicolumn{3}{|c|}{ Coordinates (Angstroms) } \\
\hline & & & $\mathrm{X}$ & $\mathrm{Y}$ & $\mathrm{z}$ \\
\hline & -2 & & 7 & --ー-ー-ー-ー & -- \\
\hline 1 & 29 & 0 & -0.000000 & 1.800000 & -0.000000 \\
\hline 2 & 29 & 0 & 0.000000 & -1.800000 & -0.000000 \\
\hline 3 & 8 & 0 & 0.700000 & 0.000000 & 0.000000 \\
\hline 4 & 8 & 0 & -0.700000 & -0.000000 & 0.000000 \\
\hline 5 & 7 & 0 & 1.414214 & 3.214214 & 0.000000 \\
\hline 6 & 7 & 0 & -1.414214 & 3.214214 & -0.000000 \\
\hline 7 & 7 & 0 & -1.414214 & -3.214214 & 0.000000 \\
\hline 8 & 7 & 0 & 1.414214 & -3.214214 & -0.000000 \\
\hline 9 & 1 & 0 & 2.320521 & 2.791595 & 0.000000 \\
\hline 10 & 1 & 0 & -2.320521 & 2.791595 & -0.000000 \\
\hline 11 & 1 & 0 & -2.320521 & -2.791595 & 0.000000 \\
\hline 12 & 1 & 0 & 2.320521 & -2.791595 & -0.000000 \\
\hline 13 & 1 & 0 & 1.323827 & 3.788290 & 0.813798 \\
\hline 14 & 1 & 0 & -1.323827 & 3.788290 & -0.813798 \\
\hline 15 & 1 & 0 & -1.323827 & -3.788290 & 0.813798 \\
\hline 16 & 1 & 0 & 1.323827 & -3.788290 & -0.813798 \\
\hline 17 & 1 & 0 & 1.323827 & 3.788290 & -0.813798 \\
\hline 18 & 1 & 0 & -1.323827 & 3.788290 & 0.813798 \\
\hline 19 & 1 & 0 & -1.323827 & -3.788290 & -0.813798 \\
\hline 20 & 1 & 0 & 1.323827 & -3.788290 & 0.813798 \\
\hline
\end{tabular}

\section{$\left\{\left(\mathrm{NH}_{3}\right)_{3} \mathrm{Cu}\right\}_{2} \mathrm{O}_{2}{ }^{2+}(\mathbf{3})$}

$0 \%$

\begin{tabular}{|c|c|c|c|c|c|}
\hline \multirow{2}{*}{$\begin{array}{l}\text { Center } \\
\text { Number }\end{array}$} & \multirow{2}{*}{$\begin{array}{l}\text { Atomic } \\
\text { Number }\end{array}$} & \multirow{2}{*}{$\begin{array}{l}\text { Atomic } \\
\text { Type }\end{array}$} & \multicolumn{3}{|c|}{ Coordinates (Angstroms) } \\
\hline & & & $\mathrm{x}$ & $\mathrm{Y}$ & $\mathrm{z}$ \\
\hline 1 & 8 & 0 & -0.003940 & 0.496472 & 0.452986 \\
\hline 2 & 8 & 0 & 0.003940 & -0.496472 & -0.452986 \\
\hline 3 & 29 & 0 & -0.003612 & 2.404409 & -0.230002 \\
\hline 4 & 29 & 0 & 0.003612 & -2.404409 & 0.230002 \\
\hline 5 & 7 & 0 & 1.190098 & 2.088821 & -1.928357 \\
\hline 6 & 7 & 0 & 0.551959 & 3.211850 & 1.640773 \\
\hline 7 & 7 & 0 & -0.551959 & -3.211850 & -1.640773 \\
\hline 8 & 7 & 0 & -1.190098 & -2.088821 & 1.928357 \\
\hline 9 & 7 & 0 & -1.660242 & 3.573354 & -0.878350 \\
\hline 10 & 7 & 0 & 1.660242 & -3.573354 & 0.878350 \\
\hline 11 & 1 & 0 & -1.386347 & 4.519536 & -1.181113 \\
\hline 12 & 1 & 0 & 1.386347 & -4.519536 & 1.181113 \\
\hline 13 & 1 & 0 & -2.371992 & 3.708222 & -0.146259 \\
\hline
\end{tabular}




$\begin{array}{rrrrrr}14 & 1 & 0 & 2.371992 & -3.708222 & 0.146259 \\ 15 & 1 & 0 & -2.157125 & 3.154410 & -1.677345 \\ 16 & 1 & 0 & 2.157125 & -3.154410 & 1.677345 \\ 17 & 1 & 0 & 2.158182 & 2.420139 & -1.812378 \\ 18 & 1 & 0 & 1.254298 & 2.622711 & 2.109147 \\ 19 & 1 & 0 & -2.158182 & -2.420139 & 1.812378 \\ 20 & 1 & 0 & -1.254298 & -2.622711 & -2.109147 \\ 21 & 1 & 0 & 0.847713 & 2.507842 & -2.803969 \\ 22 & 1 & 0 & 0.971890 & 4.149146 & 1.562455 \\ 23 & 1 & 0 & -0.847713 & -2.507842 & 2.803969 \\ 24 & 1 & 0 & -0.971890 & -4.149146 & -1.562455 \\ 25 & 1 & 0 & 1.240054 & 1.072341 & -2.084322 \\ 26 & 1 & 0 & -0.230707 & 3.305888 & 2.303239 \\ 27 & 1 & 0 & -1.240054 & -1.072341 & 2.084322 \\ 28 & 1 & 0 & 0.230707 & -3.305888 & -2.303239 \\ --------1325\end{array}$

$100 \%$

\begin{tabular}{|c|c|c|c|c|c|}
\hline \multirow{2}{*}{$\begin{array}{l}\text { Center } \\
\text { Number }\end{array}$} & \multirow{2}{*}{$\begin{array}{l}\text { Atomic } \\
\text { Number }\end{array}$} & \multirow{2}{*}{$\begin{array}{c}\text { Atomic } \\
\text { Type }\end{array}$} & \multicolumn{3}{|c|}{ Coordinates (Angstroms) } \\
\hline & & & $\mathrm{x}$ & $\mathrm{Y}$ & $\mathrm{z}$ \\
\hline 1 & 8 & 0 & 0.000000 & 0.000000 & 0.730662 \\
\hline 2 & 8 & 0 & 0.000000 & 0.000000 & -0.730662 \\
\hline 3 & 29 & 0 & -0.411730 & 1.807560 & 0.000000 \\
\hline 4 & 29 & 0 & 0.411730 & -1.807560 & 0.000000 \\
\hline 5 & 7 & 0 & 0.000000 & 3.032672 & -1.605648 \\
\hline 6 & 7 & 0 & 0.000000 & 3.032672 & 1.605648 \\
\hline 7 & 7 & 0 & 0.000000 & -3.032672 & 1.605648 \\
\hline 8 & 7 & 0 & 0.000000 & -3.032672 & -1.605648 \\
\hline 9 & 7 & 0 & -2.633478 & 1.684278 & 0.000000 \\
\hline 10 & 7 & 0 & 2.633478 & -1.684278 & 0.000000 \\
\hline 11 & 1 & 0 & -3.183738 & 2.544750 & 0.000000 \\
\hline 12 & 1 & 0 & 3.183738 & -2.544750 & 0.000000 \\
\hline 13 & 1 & 0 & -2.943994 & 1.151207 & 0.813577 \\
\hline 14 & 1 & 0 & 2.943994 & -1.151207 & -0.813577 \\
\hline 15 & 1 & 0 & -2.943994 & 1.151207 & -0.813577 \\
\hline 16 & 1 & 0 & 2.943994 & -1.151207 & 0.813577 \\
\hline 17 & 1 & 0 & 0.993345 & 3.260483 & -1.683210 \\
\hline 18 & 1 & 0 & 0.993345 & 3.260483 & 1.683210 \\
\hline 19 & 1 & 0 & -0.993345 & -3.260483 & 1.683210 \\
\hline 20 & 1 & 0 & -0.993345 & -3.260483 & -1.683210 \\
\hline 21 & 1 & 0 & -0.501839 & 3.922784 & -1.603432 \\
\hline 22 & 1 & 0 & -0.501839 & 3.922784 & 1.603432 \\
\hline 23 & 1 & 0 & 0.501839 & -3.922784 & 1.603432 \\
\hline 24 & 1 & 0 & 0.501839 & -3.922784 & -1.603432 \\
\hline 25 & 1 & 0 & -0.251567 & 2.561760 & -2.477161 \\
\hline 26 & 1 & 0 & -0.251567 & 2.561760 & 2.477161 \\
\hline 27 & 1 & 0 & 0.251567 & -2.561760 & 2.477161 \\
\hline 28 & 1 & 0 & 0.251567 & -2.561760 & -2.477161 \\
\hline
\end{tabular}


$\left\{(\text { Imid })_{3} \mathrm{Cu}\right\}_{2} \mathrm{O}_{2}{ }^{2+}(4$ BLYP $)$

$\begin{array}{rrrr}\text { bis }(\boldsymbol{\mu}-\mathbf{- o x o}) & & & \\ \mathrm{Cu} & 1.175232 & 0.760040 & -0.408035 \\ \mathrm{Cu} & -1.175232 & -0.760040 & 0.408035 \\ \mathrm{O} & -0.578995 & 0.419067 & -0.890793 \\ \mathrm{O} & 0.578995 & -0.419067 & 0.890793 \\ \mathrm{C} & 2.673782 & -0.782913 & -3.100037 \\ \mathrm{~N} & 2.309341 & -0.842834 & -1.803986 \\ \mathrm{C} & 2.373306 & -2.202637 & -1.451370 \\ \mathrm{C} & 2.780945 & -2.958145 & -2.537186 \\ \mathrm{~N} & 2.966995 & -2.043427 & -3.575989 \\ \mathrm{H} & 3.278397 & -2.279037 & -4.543488 \\ \mathrm{H} & 2.731293 & 0.118057 & -3.713440 \\ \mathrm{H} & 2.117615 & -2.528519 & -0.441818 \\ \mathrm{H} & 2.956451 & -4.027100 & -2.668716 \\ \mathrm{C} & -2.673782 & 0.782913 & 3.100037 \\ \mathrm{~N} & -2.309341 & 0.842834 & 1.803986 \\ \mathrm{C} & -2.373306 & 2.202637 & 1.451370 \\ \mathrm{C} & -2.780945 & 2.958145 & 2.537186 \\ \mathrm{~N} & -2.966995 & 2.043427 & 3.575989 \\ \mathrm{H} & -3.278397 & 2.279037 & 4.543488 \\ \mathrm{H} & -2.731293 & -0.118057 & 3.713440 \\ \mathrm{H} & -2.117615 & 2.528519 & 0.441818 \\ \mathrm{H} & -2.956451 & 4.027100 & 2.668716 \\ \mathrm{C} & 4.047394 & 0.624649 & 0.477554 \\ \mathrm{~N} & 2.827621 & 1.187576 & 0.621658 \\ \mathrm{C} & 2.874023 & 2.006958 & 1.762100 \\ \mathrm{C} & 4.141708 & 1.944336 & 2.305817 \\ \mathrm{~N} & 4.862717 & 1.071945 & 1.484300 \\ \mathrm{H} & 5.862473 & 0.798355 & 1.619736 \\ \mathrm{H} & 4.337326 & -0.080521 & -0.301849 \\ \mathrm{H} & 1.997314 & 2.559647 & 2.103485 \\ \mathrm{H} & 4.587509 & 2.424515 & 3.178909 \\ \mathrm{C} & 2.275391 & 3.217500 & -1.804626 \\ \mathrm{~N} & 1.335693 & 2.248703 & -1.716843 \\ \mathrm{C} & 0.393204 & 2.483521 & -2.729202 \\ \mathrm{C} & 0.769547 & 3.607483 & -3.437103 \\ \mathrm{~N} & 1.953979 & 4.053345 & -2.841965 \\ \mathrm{H} & 2.501053 & 4.895157 & -3.133041 \\ \mathrm{H} & 3.147446 & 3.336136 & -1.162048 \\ \mathrm{H} & -0.470383 & 1.827087 & -2.849350 \\ \mathrm{H} & 0.317505 & 4.120956 & -4.287643 \\ \mathrm{C} & -2.275391 & -3.217500 & 1.804626\end{array}$




$\begin{array}{lrrr}\mathrm{N} & -1.335693 & -2.248703 & 1.716843 \\ \mathrm{C} & -0.393204 & -2.483521 & 2.729202 \\ \mathrm{C} & -0.769547 & -3.607483 & 3.437103 \\ \mathrm{~N} & -1.953979 & -4.053345 & 2.841965 \\ \mathrm{H} & -2.501053 & -4.895157 & 3.133041 \\ \mathrm{H} & -3.147446 & -3.336136 & 1.162048 \\ \mathrm{H} & 0.470383 & -1.827087 & 2.849350 \\ \mathrm{H} & -0.317505 & -4.120956 & 4.287643 \\ \mathrm{C} & -4.047394 & -0.624649 & -0.477554 \\ \mathrm{~N} & -2.827621 & -1.187576 & -0.621658 \\ \mathrm{C} & -2.874023 & -2.006958 & -1.762100 \\ \mathrm{C} & -4.141708 & -1.944336 & -2.305817 \\ \mathrm{~N} & -4.862717 & -1.071945 & -1.484300 \\ \mathrm{H} & -5.862473 & -0.798355 & -1.619736 \\ \mathrm{H} & -4.337326 & 0.080521 & 0.301849 \\ \mathrm{H} & -1.997314 & -2.559647 & -2.103485 \\ \mathrm{H} & -4.587509 & -2.424515 & -3.178909\end{array}$

\section{side-on peroxo}

$\begin{array}{rrrr}\mathrm{Cu} & -1.511444 & -0.778793 & 0.834946 \\ \mathrm{Cu} & 1.511444 & 0.778793 & -0.834946 \\ \mathrm{O} & 0.421753 & -0.231125 & 0.544937 \\ \mathrm{O} & -0.421753 & 0.231125 & -0.544937 \\ \mathrm{C} & -2.574234 & 0.985306 & 3.292038 \\ \mathrm{~N} & -2.178909 & 0.936478 & 2.003021 \\ \mathrm{C} & -2.184418 & 2.263916 & 1.541168 \\ \mathrm{C} & -2.586563 & 3.112228 & 2.556625 \\ \mathrm{~N} & -2.828857 & 2.287537 & 3.657654 \\ \mathrm{H} & -3.150391 & 2.608154 & 4.597519 \\ \mathrm{H} & -2.679581 & 0.133728 & 3.966873 \\ \mathrm{H} & -1.893097 & 2.497772 & 0.516190 \\ \mathrm{H} & -2.722641 & 4.193924 & 2.602509 \\ \mathrm{C} & 2.574234 & -0.985306 & -3.292038 \\ \mathrm{~N} & 2.178909 & -0.936478 & -2.003021 \\ \mathrm{C} & 2.184418 & -2.263916 & -1.541168 \\ \mathrm{C} & 2.586563 & -3.112228 & -2.556625 \\ \mathrm{~N} & 2.828857 & -2.287537 & -3.657654 \\ \mathrm{H} & 3.150391 & -2.608154 & -4.597519 \\ \mathrm{H} & 2.679581 & -0.133728 & -3.966873 \\ \mathrm{H} & 1.893097 & -2.497772 & -0.516190 \\ \mathrm{H} & 2.722641 & -4.193924 & -2.602509 \\ \mathrm{C} & -4.389664 & -0.772171 & -0.115082 \\ \mathrm{~N} & -3.148636 & -1.292755 & -0.237488 \\ \mathrm{C} & -3.194458 & -2.205124 & -1.306564 \\ \mathrm{C} & -4.473541 & -2.234924 & -1.827362 \\ \mathrm{~N} & -5.211746 & -1.326691 & -1.063980 \\ \mathrm{H} & -6.224258 & -1.105560 & -1.195557 \\ & & & \end{array}$




$\begin{array}{rrrr}\mathrm{H} & -4.703537 & -0.023041 & 0.613312 \\ \mathrm{H} & -2.306198 & -2.755951 & -1.619431 \\ \mathrm{H} & -4.917816 & -2.799835 & -2.648758 \\ \mathrm{C} & -2.086868 & -3.356094 & 2.338821 \\ \mathrm{~N} & -1.290131 & -2.278030 & 2.164505 \\ \mathrm{C} & -0.235855 & -2.398605 & 3.087051 \\ \mathrm{C} & -0.401657 & -3.556671 & 3.821167 \\ \mathrm{~N} & -1.572220 & -4.146057 & 3.336258 \\ \mathrm{H} & -1.986084 & -5.043000 & 3.676025 \\ \mathrm{H} & -2.997086 & -3.582153 & 1.781647 \\ \mathrm{H} & 0.552246 & -1.646332 & 3.142044 \\ \mathrm{H} & 0.187042 & -4.007950 & 4.621811 \\ \mathrm{C} & 2.086868 & 3.356094 & -2.338821 \\ \mathrm{~N} & 1.290131 & 2.278030 & -2.164505 \\ \mathrm{C} & 0.235855 & 2.398605 & -3.087051 \\ \mathrm{C} & 0.401657 & 3.556671 & -3.821167 \\ \mathrm{~N} & 1.572220 & 4.146057 & -3.336258 \\ \mathrm{H} & 1.986084 & 5.043000 & -3.676025 \\ \mathrm{H} & 2.997086 & 3.582153 & -1.781647 \\ \mathrm{H} & -0.552246 & 1.646332 & -3.142044 \\ \mathrm{H} & -0.187042 & 4.007950 & -4.621811 \\ \mathrm{C} & 4.389664 & 0.772171 & 0.115082 \\ \mathrm{~N} & 3.148636 & 1.292755 & 0.237488 \\ \mathrm{C} & 3.194458 & 2.205124 & 1.306564 \\ \mathrm{C} & 4.473541 & 2.234924 & 1.827362 \\ \mathrm{~N} & 5.211746 & 1.326691 & 1.063980 \\ \mathrm{H} & 6.224258 & 1.105560 & 1.195557 \\ \mathrm{H} & 4.703537 & 0.023041 & -0.613312 \\ \mathrm{H} & 2.306198 & 2.755951 & 1.619431 \\ \mathrm{H} & 4.917816 & 2.799835 & 2.648758\end{array}$

\section{end-on peroxo}

$\begin{array}{rrrr}\mathrm{Cu} & -1.751587 & -1.499634 & -0.702774 \\ \mathrm{Cu} & 1.751587 & 1.499634 & 0.702774 \\ \mathrm{O} & 0.594406 & -0.112076 & 0.274124 \\ \mathrm{O} & -0.594406 & 0.112076 & -0.274124 \\ \mathrm{C} & -1.035980 & -3.750671 & -2.650436 \\ \mathrm{~N} & -0.590317 & -2.756317 & -1.851852 \\ \mathrm{C} & 0.807755 & -2.729034 & -1.989197 \\ \mathrm{C} & 1.203979 & -3.714096 & -2.873611 \\ \mathrm{~N} & 0.028381 & -4.349335 & -3.280884 \\ \mathrm{H} & -0.031555 & -5.144531 & -3.954697 \\ \mathrm{H} & -2.076309 & -4.049561 & -2.791367 \\ \mathrm{H} & 1.408218 & -2.000443 & -1.441757 \\ \mathrm{H} & 2.184082 & -4.018585 & -3.243286 \\ \mathrm{C} & 1.035980 & 3.750671 & 2.650436 \\ \mathrm{~N} & 0.590317 & 2.756317 & 1.851852\end{array}$




\begin{tabular}{|c|c|c|c|}
\hline C & -0.807755 & 2.729034 & 1.989197 \\
\hline C & -1.203979 & 3.714096 & 2.873611 \\
\hline $\mathrm{N}$ & -0.028381 & 4.349335 & 3.280884 \\
\hline $\mathrm{H}$ & 0.031555 & 5.144531 & 3.954697 \\
\hline $\mathrm{H}$ & 2.076309 & 4.049561 & 2.791367 \\
\hline $\mathrm{H}$ & -1.408218 & 2.000443 & 1.441757 \\
\hline $\mathrm{H}$ & -2.184082 & 4.018585 & 3.243286 \\
\hline $\mathrm{C}$ & -4.455032 & -0.754211 & -1.969803 \\
\hline $\mathrm{N}$ & -3.178589 & -0.402710 & -1.692886 \\
\hline C & -2.959824 & 0.829453 & -2.332809 \\
\hline $\mathrm{C}$ & -4.107651 & 1.221436 & -2.993042 \\
\hline $\mathrm{N}$ & -5.041153 & 0.209808 & -2.754364 \\
\hline $\mathrm{H}$ & -6.020828 & 0.188965 & -3.115768 \\
\hline $\mathrm{H}$ & -4.963440 & -1.660324 & -1.634506 \\
\hline $\mathrm{H}$ & -1.993134 & 1.330719 & -2.266068 \\
\hline $\mathrm{H}$ & -4.344100 & 2.098053 & -3.597946 \\
\hline C & -3.304108 & -2.203217 & 1.845093 \\
\hline $\mathrm{N}$ & -2.340302 & -2.538895 & 0.957748 \\
\hline C & -1.801376 & -3.760788 & 1.400360 \\
\hline $\mathrm{C}$ & -2.445908 & -4.160965 & 2.554230 \\
\hline $\mathrm{N}$ & -3.389832 & -3.165771 & 2.820800 \\
\hline $\mathrm{H}$ & -4.055771 & -3.161804 & 3.625275 \\
\hline $\mathrm{H}$ & -3.927612 & -1.307922 & 1.810760 \\
\hline $\mathrm{H}$ & -0.998398 & -4.254440 & 0.852051 \\
\hline $\mathrm{H}$ & -2.328018 & -5.036118 & 3.195251 \\
\hline $\mathrm{C}$ & 3.304108 & 2.203217 & -1.845093 \\
\hline $\mathrm{N}$ & 2.340302 & 2.538895 & -0.957748 \\
\hline $\mathrm{C}$ & 1.801376 & 3.760788 & -1.400360 \\
\hline C & 2.445908 & 4.160965 & -2.554230 \\
\hline $\mathrm{N}$ & 3.389832 & 3.165771 & -2.820800 \\
\hline $\mathrm{H}$ & 4.055771 & 3.161804 & -3.625275 \\
\hline $\mathrm{H}$ & 3.927612 & 1.307922 & -1.810760 \\
\hline $\mathrm{H}$ & 0.998398 & 4.254440 & -0.852051 \\
\hline $\mathrm{H}$ & 2.328018 & 5.036118 & -3.195251 \\
\hline C & 4.455032 & 0.754211 & 1.969803 \\
\hline $\mathrm{N}$ & 3.178589 & 0.402710 & 1.692886 \\
\hline C & 2.959824 & -0.829453 & 2.332809 \\
\hline C & 4.107651 & -1.221436 & 2.993042 \\
\hline $\mathrm{N}$ & 5.041153 & -0.209808 & 2.754364 \\
\hline $\mathrm{H}$ & 6.020828 & -0.188965 & 3.115768 \\
\hline $\mathrm{H}$ & 4.963440 & 1.660324 & 1.634506 \\
\hline $\mathrm{H}$ & 1.993134 & -1.330719 & 2.266068 \\
\hline $\mathrm{H}$ & 4.344100 & -2.098053 & 3.597946 \\
\hline
\end{tabular}


Singlet and Broken-symmetry Absolute $\left(E_{h}\right)$ and Relative $\left(\mathrm{kcal} \mathrm{mol}^{-1}\right)$ Electronic Energies

$\mathrm{Cu}_{2} \mathrm{O}_{2}{ }^{2+}(\mathbf{0})$

\begin{tabular}{|c|c|c|c|c|c|c|}
\hline & & & omer & & & \\
\hline & $F=0$ & $F=20$ & $F=40$ & $F=60$ & $F=80$ & $F=100$ \\
\hline $\mathrm{HF}$ & -541.29316 & -541.31416 & -541.30641 & -541.28090 & -541.25118 & -541.21734 \\
\hline MP2 & -542.18261 & -542.17751 & -542.16252 & -542.14118 & -542.15120 & -542.17675 \\
\hline CCSD & -542.10730 & -542.11182 & -542.09936 & -542.07698 & -542.06711 & -542.06620 \\
\hline $\operatorname{CCSD}(T)$ & -542.15999 & -542.15992 & -542.14611 & -542.12449 & -542.12810 & -542.13733 \\
\hline $\operatorname{CR}-\operatorname{CCSD}(\mathrm{T})$ & -542.14183 & -542.14386 & -542.13064 & -542.10858 & -542.10732 & -542.11221 \\
\hline $\operatorname{CR}-\mathrm{CC}(2,3), A$ & -542.16082 & -542.16186 & -542.14830 & -542.12652 & -542.12790 & -542.13304 \\
\hline$C R-C C(2,3), D$ & -542.16304 & -542.16410 & -542.15072 & -542.12911 & -542.13051 & -542.13669 \\
\hline $\operatorname{CCSD}(\mathrm{TQ})$ & -542.14877 & -542.14851 & -542.13474 & -542.11284 & -542.11145 & -542.12244 \\
\hline CR-CCSD(TQ) & -542.14773 & -542.14903 & -542.13564 & -542.11380 & -542.11322 & -542.11873 \\
\hline $\mathrm{CR}-\mathrm{CC}(2,3)+\mathrm{Q}^{a}$ & -542.16894 & -542.16927 & -542.15572 & -542.13434 & -542.13641 & -542.14321 \\
\hline $\operatorname{CAS}(16,14)$ & -541.50274 & -541.50200 & -541.48831 & -541.46466 & -541.45012 & -541.42189 \\
\hline CASPT2 $(16,14)$ & -542.40875 & -542.40688 & -542.39254 & -542.37837 & -542.39108 & -542.39664 \\
\hline MSCASPT2 $(16,14)$ & -542.43252 & -542.42741 & -542.41094 & -542.39047 & -542.39674 & -542.39670 \\
\hline bs-BLYP & -544.25169 & -544.24859 & -544.23566 & -544.22283 & -544.21738 & -544.21183 \\
\hline bs-B3LYP & -544.31860 & -544.32209 & -544.31227 & -544.29986 & -544.29095 & -544.27844 \\
\hline bs-mPWPW91 & -544.50921 & -544.50848 & -544.49691 & -544.48500 & -544.47984 & -544.47385 \\
\hline bs-mPW1PW91 & -544.28720 & -544.29393 & -544.28556 & -544.27345 & -544.26345 & -544.24839 \\
\hline bs-MPW1K & -544.14230 & -544.15407 & -544.14712 & -544.13345 & -544.11860 & -544.09546 \\
\hline bs-TPSS & -544.20265 & -544.20137 & -544.19021 & -544.17973 & -544.17621 & -544.17232 \\
\hline bs-TPSSh & -544.13302 & -544.13481 & -544.12502 & -544.11452 & -544.10911 & -544.10160 \\
\hline BLYP/BS1d & -3430.97210 & & & & & -3430.92225 \\
\hline BLYP/BS1t & -3430.98511 & & & & & -3430.93309 \\
\hline
\end{tabular}

a Computed as CR-CC(2,3),D + \{CR-CCSD(TQ) $-\mathrm{CR}-\mathrm{CCSD}(\mathrm{T})\}$ 


\begin{tabular}{|c|c|c|c|c|c|c|}
\hline & $F=0$ & $F=20$ & $\begin{array}{l}\text { Isomer } \\
F=40\end{array}$ & $F=60$ & $F=80$ & $F=100$ \\
\hline $\mathrm{HF}$ & -47.6 & -60.8 & -55.9 & -39.9 & -21.2 & 0.0 \\
\hline MP2 & -3.7 & -0.5 & 8.9 & 22.3 & 16.0 & 0.0 \\
\hline CCSD & -25.8 & -28.6 & -20.8 & -6.8 & -0.6 & 0.0 \\
\hline $\operatorname{CCSD}(T)$ & -14.2 & -14.2 & -5.5 & 8.1 & 5.8 & 0.0 \\
\hline $\operatorname{CR}-\operatorname{CCSD}(T)$ & -18.6 & -19.9 & -11.6 & 2.3 & 3.1 & 0.0 \\
\hline CR-CC $(2,3), A$ & -17.4 & -18.1 & -9.6 & 4.1 & 3.2 & 0.0 \\
\hline CR-CC $(2,3), D$ & -16.5 & -17.2 & -8.8 & 4.8 & 3.9 & 0.0 \\
\hline $\operatorname{CCSD}(\mathrm{TQ})$ & -16.5 & -16.4 & -7.7 & 6.0 & 6.9 & 0.0 \\
\hline CR-CCSD(TQ) & -18.2 & -19.0 & -10.6 & 3.1 & 3.5 & 0.0 \\
\hline $\mathrm{CR}-\mathrm{CC}(2,3)+\mathrm{Q}^{a}$ & -16.2 & -16.4 & -7.9 & 5.6 & 4.3 & 0.0 \\
\hline $\operatorname{CAS}(16,14)$ & -50.7 & -50.3 & -41.7 & -26.8 & -17.7 & 0.0 \\
\hline CASPT2 $(16,14)$ & -7.6 & -6.4 & 2.6 & 11.5 & 3.5 & 0.0 \\
\hline $\operatorname{MSCASPT2}(16,14)$ & -22.5 & -19.3 & -8.9 & 3.9 & 0.0 & 0.0 \\
\hline bs-BLYP & -25.0 & -23.1 & -15.0 & -6.9 & -3.5 & 0.0 \\
\hline bs-B3LYP & -25.2 & -27.4 & -21.2 & -13.4 & -7.8 & 0.0 \\
\hline bs-mPWPW91 & -22.2 & -21.7 & -14.5 & -7.0 & -3.8 & 0.0 \\
\hline bs-mPW1PW91 & -24.4 & -28.6 & -23.3 & -15.7 & -9.5 & 0.0 \\
\hline bs-MPW1K & -29.4 & -36.8 & -32.4 & -23.8 & -14.5 & 0.0 \\
\hline bs-TPSS & -19.0 & -18.2 & -11.2 & -4.7 & -2.4 & 0.0 \\
\hline bs-TPSSh & -19.7 & -20.8 & -14.7 & -8.1 & -4.7 & 0.0 \\
\hline sumbs-BLYP & -21.9 & -19.5 & -10.3 & -1.6 & 0.2 & 0.0 \\
\hline sumbs-B3LYP & -21.4 & -23.2 & -16.1 & -7.5 & -3.2 & 0.0 \\
\hline sumbs-mPWPW91 & -18.9 & -18.1 & -9.8 & -1.8 & -0.2 & 0.0 \\
\hline sumbs-mPW1PW91 & -21.0 & -24.8 & -18.7 & -10.2 & -4.9 & 0.0 \\
\hline sumbs-mPW1K & -27.7 & -34.9 & -29.7 & -19.8 & -10.3 & 0.0 \\
\hline sumbs-TPSS & -15.4 & -14.1 & -6.2 & 0.6 & 1.1 & 0.0 \\
\hline sumbs-TPSSh & -15.8 & -16.4 & -9.5 & -2.5 & -0.6 & 0.0 \\
\hline BLYP/BS1d & -31.3 & & & & & 0.0 \\
\hline BLYP/BS1t & -32.6 & & & & & 0.0 \\
\hline
\end{tabular}


${ }^{a}$ Computed as CR-CC(2,3),D $+\{\mathrm{CR}-\mathrm{CCSD}(\mathrm{TQ})-\mathrm{CR}-\mathrm{CCSD}(\mathrm{T})\}$

$\left\{\left(\mathrm{NH}_{3}\right) \mathrm{Cu}\right\}_{2} \mathrm{O}_{2}{ }^{2+}(\mathbf{1})$

\begin{tabular}{|c|c|c|c|c|c|c|}
\hline \multirow{2}{*}{\multicolumn{7}{|c|}{$\begin{array}{l}\text { Isomer } \\
\qquad F=40\end{array}$}} \\
\hline & $F=0$ & $F=20$ & $F=40$ & $F=60$ & $F=80$ & $F=100$ \\
\hline $\mathrm{HF}$ & -653.89295 & -653.89304 & -653.88182 & -653.86752 & -653.86148 & -653.85236 \\
\hline MP2 & -655.24152 & -655.24325 & -655.23572 & -655.24802 & -655.26946 & -655.29541 \\
\hline CCSD & -655.18358 & -655.18577 & -655.17776 & -655.16849 & -655.17598 & -655.18490 \\
\hline $\operatorname{CCSD}(T)$ & -655.25938 & -655.26337 & -655.25777 & -655.25769 & -655.26771 & -655.28025 \\
\hline $\operatorname{CR}-\operatorname{CCSD}(T)$ & -655.22488 & -655.22755 & -655.22016 & -655.21764 & -655.22709 & -655.23810 \\
\hline CR-CC $(2,3), A$ & -655.26179 & -655.26575 & -655.25945 & -655.25352 & -655.26310 & -655.27333 \\
\hline CR-CC $(2,3), D$ & -655.26200 & -655.26608 & -655.25983 & -655.25570 & -655.26605 & -655.27575 \\
\hline $\operatorname{CCSD}(\mathrm{TQ})$ & -655.23562 & -655.23649 & -655.22769 & -655.23397 & -655.24572 & -655.26114 \\
\hline CR-CCSD (TQ) & -655.23166 & -655.23469 & -655.22773 & -655.22348 & -655.23307 & -655.24414 \\
\hline $\mathrm{CR}-\mathrm{CC}(2,3), \mathrm{D}+$ & -655.26877 & -655.27321 & -655.26739 & -655.26154 & -655.27203 & -655.28178 \\
\hline $\operatorname{CAS}(16,14), \mathrm{Ci}$ & -654.06111 & & & & & -654.21506 \\
\hline PT2 $(16,14), \mathrm{Ci}$ & -655.21078 & & & & & -655.18470 \\
\hline MSPT2(16,14),Ci & -655.21126 & & & & & -655.18470 \\
\hline PT2 $(12,12)$ 4root & -655.16717 & -655.18992 & -655.13556 & -655.14457 & -655.16007 & -655.18524 \\
\hline $\operatorname{MS}(12,12) 4$ root & -655.18276 & -655.19553 & -655.16374 & -655.17622 & -655.18803 & -655.19639 \\
\hline PT2 $(12,12)$ 5root & -655.18383 & -655.15752 & -655.14292 & -655.15418 & -655.16430 & -655.19701 \\
\hline MS $(12,12)$ root & -655.19654 & -655.19201 & -655.18218 & -655.18724 & -655.20506 & -655.23061 \\
\hline $\operatorname{MS}(12,12) 6$ root & -655.20535 & -655.19027 & -655.17372 & -655.18888 & -655.19786 & -655.22398 \\
\hline PT2 $(12,14) 6$ root & -655.15861 & -655.14574 & -655.16148 & -655.16662 & -655.15478 & -655.19735 \\
\hline $\operatorname{MS}(12,14) 6$ root & -655.19466 & -655.17673 & -655.18130 & -655.18249 & -655.17550 & -655.22728 \\
\hline PT2 $(16,14) 4$ root & -655.11383 & -655.19661 & -655.13940 & -655.15044 & -655.16995 & -655.19309 \\
\hline $\operatorname{MS}(16,14) 4$ root & -655.14104 & -655.20092 & -655.17959 & -655.19378 & -655.21299 & -655.20140 \\
\hline bs-BLYP & -657.60831 & -657.61162 & -657.60773 & -657.60024 & -657.59473 & -657.59109 \\
\hline bs-B3LYP & -657.71802 & -657.72257 & -657.72018 & -657.71384 & -657.70826 & -657.70433 \\
\hline bs-mPWPW91 & -657.89622 & -657.90084 & -657.89821 & -657.89187 & -657.88738 & -657.88501 \\
\hline
\end{tabular}




\begin{tabular}{|c|c|c|c|c|c|c|}
\hline bs-mPW1PW91 & -657.64379 & -657.64924 & -657.64782 & -657.64230 & -657.63712 & -657.63334 \\
\hline bs-MPW1K & -657.47913 & -657.48434 & -657.48273 & -657.47666 & -657.46994 & -657.46409 \\
\hline S-TPSS & -657.60969 & -657.61430 & -657.61215 & -657.60684 & -657.60375 & 60297 \\
\hline S-TPSSh & -657.52202 & -657.52707 & -657.52549 & -657.52055 & -657.51715 & -657.51576 \\
\hline LYP/BS1d & -3544.31882 & & & & & -3544 \\
\hline YP/BS1t & -3544.33547 & & & & & -3544. \\
\hline
\end{tabular}

${ }^{a}$ Computed as CR-CC(2,3),D $+\{\mathrm{CR}-\mathrm{CCSD}(\mathrm{TQ})-\mathrm{CR}-\mathrm{CCSD}(\mathrm{T})\}$

\begin{tabular}{|c|c|c|c|c|c|c|}
\hline \multicolumn{7}{|c|}{ Isomer } \\
\hline & $F=0$ & $F=20$ & $F=40$ & $F=60$ & $F=80$ & $F=100$ \\
\hline $\mathrm{HF}$ & -25.5 & -25.5 & -18.5 & -9.5 & -5.7 & 0.0 \\
\hline MP2 & 33.8 & 32.7 & 37.5 & 29.7 & 16.3 & 0.0 \\
\hline CCSD & 0.8 & -0.5 & 4.5 & 10.3 & 5.6 & 0.0 \\
\hline $\operatorname{CcSD}(T)$ & 13.1 & 10.6 & 14.1 & 14.2 & 7.9 & 0.0 \\
\hline $\operatorname{CR}-\operatorname{CCSD}(T)$ & 8.3 & 6.6 & 11.3 & 12.8 & 6.9 & 0.0 \\
\hline CR-CC $(2,3), A$ & 7.2 & 4.8 & 8.7 & 12.4 & 6.4 & 0.0 \\
\hline CR-CC $(2,3), D$ & 8.6 & 6.1 & 10.0 & 12.6 & 6.1 & 0.0 \\
\hline $\operatorname{CCSD}(\mathrm{TQ})$ & 16.0 & 15.5 & 21.0 & 17.1 & 9.7 & 0.0 \\
\hline CR-CCSD (TQ) & 7.8 & 5.9 & 10.3 & 13.0 & 6.9 & 0.0 \\
\hline $\mathrm{CR}-\mathrm{CC}(2,3), \mathrm{D}+\mathrm{Q}^{a}$ & 8.2 & 5.4 & 9.0 & 12.7 & 6.1 & 0.0 \\
\hline $\operatorname{CAS}(16,14), \mathrm{Ci}$ & 96.6 & & & & & 0.0 \\
\hline PT2 $(16,14), \mathrm{Ci}$ & -16.4 & & & & & 0.0 \\
\hline MSPT2 $(16,14), \mathrm{Ci}$ & -16.7 & & & & & 0.0 \\
\hline PT2 $(12,12)$ 4root & 11.3 & -2.9 & 31.2 & 25.5 & 15.8 & 0.0 \\
\hline $\operatorname{MS}(12,12)$ 4root & 8.6 & 0.5 & 20.5 & 12.7 & 5.2 & 0.0 \\
\hline PT2 $(12,12) 5$ root & 8.3 & 24.8 & 33.9 & 26.9 & 20.5 & 0.0 \\
\hline $\operatorname{MS}(12,12)$ 5root & 21.4 & 24.2 & 30.4 & 27.2 & 16.0 & 0.0 \\
\hline $\operatorname{MS}(12,12) 6$ root & 11.7 & 21.2 & 31.5 & 22.0 & 16.4 & 0.0 \\
\hline PT2 $(12,14) 6$ root & 24.3 & 32.4 & 22.5 & 19.3 & 26.7 & 0.0 \\
\hline $\operatorname{MS}(12,14) 6$ root & 20.5 & 31.7 & 28.8 & 28.1 & 32.5 & 0.0 \\
\hline PT2 $(16,14)$ 4root & 49.7 & -2.2 & 33.7 & 26.8 & 14.5 & 0.0 \\
\hline
\end{tabular}




\begin{tabular}{|c|c|c|c|}
\hline MS $(16,14) 4$ root & 37.9 & 0.3 & 13.7 \\
\hline BLYP & -10.8 & -12.9 & -10.4 \\
\hline bs-BLYP & -8.6 & -11.4 & -10.0 \\
\hline B3LYP & -7.0 & -9.9 & -8.3 \\
\hline bs-B3LYP & -6.6 & -10.0 & -9.1 \\
\hline mPWPW91 & -9.4 & -12.7 & -11.7 \\
\hline bs-mPWPW91 & -4.2 & -7.1 & -5.8 \\
\hline mPW1PW91 & -3.9 & -7.1 & -6.1 \\
\hline bs-mPW1PW91 & -6.6 & -8.1 & -4.7 \\
\hline MPW1K & -1.0 & -2.8 & -0.3 \\
\hline bs-MPW1K & -2.7 & -5.1 & -2.5 \\
\hline TPSS & 0.0 & -2.2 & -0.2 \\
\hline bs-TPSS & -4.0 & -5.5 & -2.6 \\
\hline TPSSh & 0.7 & -1.6 & 0.5 \\
\hline bs-TPSSh & 2.2 & -0.1 & 1.5 \\
\hline BLYP/BS1d & -11.0 & & \\
\hline BLYP/BS1t & -11.7 & & \\
\hline
\end{tabular}

4.8
-5.7
-6.0
-4.3
-5.6
-7.9
-2.4
-3.0
0.0
3.1
1.3
2.7
1.4
3.3
3.9

$$
-7.3
$$

${ }^{a}$ Computed as CR-CC(2,3),D $+\{\mathrm{CR}-\mathrm{CCSD}(\mathrm{TQ})-\mathrm{CR}-\mathrm{CCSD}(\mathrm{T})\}$

$\left\{\left(\mathrm{NH}_{3}\right)_{2} \mathrm{Cu}\right\}_{2} \mathrm{O}_{2}{ }^{2+}(\mathbf{2})$

\begin{tabular}{|c|c|c|c|c|c|c|}
\hline & $F=0$ & $F=20$ & $\begin{array}{l}\text { Isomer } \\
\qquad F=40\end{array}$ & $F=60$ & $F=80$ & $F=100$ \\
\hline $\mathrm{HF}$ & -766.45428 & -766.40311 & -766.32711 & -766.31845 & -766.37697 & -766.41615 \\
\hline MP2 & -768.24927 & -768.23683 & -768.20070 & -768.22865 & -768.30054 & -768.33506 \\
\hline CCSD & -768.20727 & -768.17796 & -768.12245 & -768.12883 & -768.19256 & -768.22608 \\
\hline $\operatorname{CCSD}(\mathrm{T})$ & -768.29984 & -768.28365 & -768.23335 & -768.24238 & -768.30626 & -768.34021 \\
\hline $\operatorname{CR}-\operatorname{CCSD}(\mathrm{T})$ & -768.24557 & -768.22395 & -768.17622 & -768.18523 & -768.25001 & -768.28393 \\
\hline CR-CC $(2,3), A$ & -768.29887 & -768.28328 & -768.23062 & -768.23574 & -768.29671 & -768.32835 \\
\hline CR-CC $(2,3), D$ & -768.29908 & -768.28137 & -768.23111 & -768.23793 & -768.30007 & -768.33235 \\
\hline $\operatorname{CAS}(14,13)$ & -766.62137 & & & & & -766.73765 \\
\hline
\end{tabular}




\begin{tabular}{|c|c|c|c|c|c|c|}
\hline CASPT2 $(14,13)$ & -768.41777 & & & & & -768.41612 \\
\hline MSPT2 $(14,13)$ & -768.420284 & & & & & -768.41612 \\
\hline bs-BLYP & -770.87738 & -770.84603 & -770.78654 & -770.77795 & -770.82747 & -770.8534 \\
\hline bs-B3LYP & -771.04820 & -771.01812 & -770.96474 & -770.95080 & -771.00783 & -77 \\
\hline s-mPWPW91 & -771.20433 & -771.17935 & -771.12490 & -771.11749 & -771.16440 & -771 \\
\hline PW1PW91 & -770.94250 & -770.91632 & -770.86791 & -770.85117 & -770.89882 & -770 \\
\hline MPW1K & -770.77202 & -770.74363 & -770.69827 & -770.67397 & -770.75944 & -770.79 \\
\hline bs-TPSS & -770.94271 & -770.91723 & -770.86286 & -770.85651 & -770.90687 & -770.933 \\
\hline bs-TPSSh & -770.84474 & -770.81896 & -770.76711 & -770.75735 & -770.80755 & -770.837 \\
\hline
\end{tabular}

\begin{tabular}{lrrrrrr}
\hline & \multicolumn{7}{c}{ Isomer } \\
HF & $F=0$ & $F=20$ & $F=40$ & $F=60$ & $F=80$ & $F=100$ \\
MP2 & -23.9 & 8.2 & 55.9 & 61.3 & 24.6 & 0.0 \\
CCSD & 53.8 & 61.6 & 84.3 & 66.8 & 21.7 & 0.0 \\
CCSD(T) & 11.8 & 30.2 & 65.0 & 61.0 & 21.0 & 0.0 \\
CR-CCSD(T) & 25.3 & 35.5 & 67.1 & 61.4 & 21.3 & 0.0 \\
CR-CC(2,3),A & 24.1 & 37.6 & 67.6 & 61.9 & 21.3 & 0.0 \\
CR-CC(2,3),D & 18.5 & 28.3 & 61.3 & 58.1 & 19.9 & 0.0 \\
CAS(14,13) & 20.9 & 32.0 & 63.5 & 59.3 & 20.3 & 0.0 \\
CASPT2(14,13) & 73.0 & & & & & 0.0 \\
MSPT2(14,13) & -1.0 & & & & & 0.0 \\
bs-BLYP & -2.6 & & & & & 0.0 \\
bs-B3LYP & -15.0 & 4.7 & 42.0 & 47.4 & 16.3 & 0.0 \\
bs-mPWPW91 & -7.2 & 11.7 & 45.2 & 54.0 & 18.2 & 0.0 \\
bs-mPW1PW91 & -10.6 & 5.1 & 39.2 & 43.9 & 14.4 & 0.0 \\
bs-MPW1K & -1.2 & 15.3 & 45.6 & 56.1 & 26.2 & 0.0 \\
bs-TPSS & 13.1 & 30.9 & 59.4 & 74.6 & 21.0 & 0.0 \\
bs-TPSSh & -5.7 & 10.2 & 44.4 & 48.3 & 16.7 & 0.0 \\
sumbs-BLYP & -4.4 & 11.8 & 44.3 & 50.5 & 19.0 & 0.0 \\
sumbs-B3LYP & -9.9 & 8.8 & 44.4 & 46.4 & 16.3 & 0.0 \\
sumbs-mPWPW91 & 7.9 & 26.0 & 56.0 & 62.3 & 18.6 & 0.0 \\
& -5.4 & 9.3 & 41.8 & 43.0 & 12.7 & 0.0
\end{tabular}




\begin{tabular}{|c|c|c|c|c|c|c|}
\hline sumbs-mPW1PW91 & 15.1 & 31.1 & 57.0 & 65.8 & 33.2 & 0.0 \\
\hline sumbs-mPW1K & 30.5 & 48.6 & 70.4 & 86.5 & 8.6 & 0.0 \\
\hline sumbs-TPSS & -0.9 & 14.2 & 46.8 & 47.5 & 16.7 & 0.0 \\
\hline sumbs-TPSSh & 6.6 & 22.1 & 52.1 & 55.3 & 23.2 & 0.0 \\
\hline
\end{tabular}

$\left\{\left(\mathrm{NH}_{3}\right)_{3} \mathrm{Cu}\right\}_{2} \mathrm{O}_{2}{ }^{2+}(\mathbf{3})$

Data in blue are with BS2, data in black are with BS1, data in green are extrapolated assuming changes computed with BS2 can be added to values computed with BS1.

\begin{tabular}{|c|c|c|c|c|c|c|}
\hline & & & Isomer & & & \\
\hline & & & & $F=60$ & $=80$ & $F=100$ \\
\hline $\mathrm{HF}$ & -878.80279 & -878.80073 & -878.78427 & -878.78494 & -878.79978 & -878.80284 \\
\hline MP2 & -880.85302 & -880.84428 & -880.83355 & -880.86420 & -880.91767 & -880.95111 \\
\hline CCSD & -880.82656 & -880.82284 & -880.80474 & -880.81926 & -880.85140 & -880.86767 \\
\hline $\operatorname{CCSD}(\mathrm{T})$ & -880.94363 & -880.93590 & -880.91314 & -880.92412 & -880.95953 & -880.98033 \\
\hline $\operatorname{CR}-\operatorname{CCSD}(\mathrm{T})$ & -880.87502 & -880.86932 & -880.84890 & -880.86677 & -880.90113 & -880.91899 \\
\hline CR-CC $(2,3), A$ & -880.93070 & -880.92485 & -880.90415 & -880.91715 & -880.94848 & -880.96434 \\
\hline CR-CC $(2,3), D$ & -880.93141 & -880.92566 & -880.90509 & -880.92075 & -880.95278 & -880.96931 \\
\hline $\mathrm{HF}$ & -878.90467 & -878.90169 & -878.88509 & -878.88590 & -878.90120 & 90729 \\
\hline MP2 & -881.18287 & -881.17356 & -881.16306 & -881.19321 & -881.24666 & 8450 \\
\hline CCSD & -881.1 & -881.1 & -881 & -881 & -881 & 9207 \\
\hline $\operatorname{CCSD}(\mathrm{T})$ & -881.29220 & -881.28162 & -881.25707 & -881.26944 & -881.30454 & -881.32953 \\
\hline $\operatorname{CR}-\operatorname{CCSD}(\mathrm{T})$ & -881.20550 & -881.19912 & -881.18074 & -881.19729 & -881.23178 & -881.25391 \\
\hline CR-CC $(2,3), A$ & -881.26119 & -881.25466 & -881.23599 & -881.24767 & -881.27913 & -881.29926 \\
\hline CR-CC( $(2,3), D$ & -881.26189 & -881.25547 & -881.23693 & -881.25127 & -881.28343 & -881.30423 \\
\hline $\operatorname{CAS}(16,14)$ & -879.15476 & & & & & -879.31259 \\
\hline PT2 $(16,14)$ & -881.30461 & & & & & -881.36746 \\
\hline MSPT2 $(16,14)$ & -881.3 & & & & & 36747 \\
\hline bs-BLYP & -884.05543 & 173 & -884 & 05 & 537 & -884.04984 \\
\hline bs-B3LYP & -884.27544 & -884.26785 & -884.24804 & -884.24505 & -884.26287 & -884.28924 \\
\hline
\end{tabular}


bs-mPWPW91 bs-mPW1PW91 bs-MPW1K bs-TPSS bs-TPSSh BLYP/BS1d BLYP/BS1t

HF

MP2

CCSD

$\operatorname{CCSD}(T)$

CR-CCSD $(T)$

CR-CC $(2,3)$,A

CR-CC $(2,3)$, D

$\mathrm{HF}$

MP2

CCSD

$\operatorname{CCSD}(T)$

$\operatorname{CR}-\operatorname{CCSD}(T)$

CR-CC $(2,3), A$

CR-CC $(2,3)$,D

$\operatorname{CAS}(16,14)$

PT2 $(16,14)$

MSPT2 $(16,14)$

bs-BLYP

bs-B3LYP

bs-mPWPW91

bs-mPW1PW91

bs-MPW1K
$-884.41862$ $-884.13334$

$-883.94614$

$-884.18636$

$-884.07245$ $-3770.76827$ $-3770.78360$

$-884.41004$ $-884.13070$ $-883.94773$ $-884.17757$

$-884.06598$

$-884.39031$ $-884.11400$ $-883.93306$ $-884.15813$ $-884.04768$

$-884.38715$ $-884.11116$ $-883.92936$ $-884.15631$ $-884.04610$

$-884.40503$ $-884.12613$ $-883.94086$ $-884.17612$ $-884.06457$

$$
F=0
$$

0.0

61.6

25.8

23.0

27.6

21.1

23.8

1.6

63.8

27.4

23.4

30.4

23.9

26.6

99.0

39.4

38.7

$-3.5$

8.7

$-1.4$

14.8

35.7

$F=20$
1.3
67.0
28.1
27.9
31.2
24.8
27.4
3.5
69.6
30.4
30.1
34.4
28.0
30.6


5.1
13.4
4.0
16.4
34.7

Isome

$$
F=40
$$

11.7

73.8

39.5

42.2

44.0

37.8

40.3

13.9

76.2

41.3

45.5

45.9

39.7

42.2

$F=60$

11.2

54.5

30.4

35.3

32.8

29.6

30.5

13.4

57.3

32.8

37.7

35.5

32.4

33.2

19.6

25.9

16.3

26.9

43.9
$-884.41636$ $-884.15688$

$-884.00298$

$-884.18972$

$-884.08246$

$-3770.76190$

$-3770.77628$

$\begin{array}{rr}F=80 & F=100 \\ 1.9 & 0.0 \\ 21.0 & 0.0 \\ 10.2 & 0.0 \\ 13.1 & 0.0 \\ 11.2 & 0.0 \\ 10.0 & 0.0 \\ 10.4 & 0.0 \\ 3.8 & 0.0 \\ 23.7 & 0.0 \\ 12.8 & 0.0 \\ 15.7 & 0.0 \\ 13.9 & 0.0 \\ 12.6 & 0.0 \\ 13.1 & 0.0 \\ & 0.0 \\ & 0.0 \\ 9.1 & 0.0 \\ 16.5 & 0.0 \\ 7.1 & 0.0 \\ 19.3 & 0.0 \\ 39.0 & 0.0 \\ & 0.0\end{array}$


bs-TPSS

bs-TPSSh

$$
2.1
$$

6.3

sumbs-BLYP

1.0

sumbs-B3LYP

22.9

sumbs-mPWPW91

3.1

sumbs-mPW1PW91

30.3

sumbs-mPW1K

53.9

7.1

17.4

sumbs-TPSSh

$-4.0$

BLYP/DZ/allelec

$-4.6$

$\begin{array}{rr}7.6 & 19.8 \\ 10.3 & 21.8 \\ 9.8 & 24.1 \\ 27.8 & 39.5 \\ 8.6 & 20.9 \\ 31.9 & 41.7 \\ 52.4 & 60.7 \\ 12.8 & 24.8 \\ 21.6 & 32.5\end{array}$

21.0

8.5

11.2

22.8
23.1

37.6

19.8

39.9

59.6

23.0

BLYP/TZ/allelec

0.0

0.0

0.0

0.0

0.0

0.0

0.0

0.0

0.0

0.0

0.0

Triplet Electronic Energies $\left(E_{h}\right)$ and $<S^{2}>$ Values (for Computing Sum-bs Energies)

$\mathrm{Cu}_{2} \mathrm{O}_{2}{ }^{2+}(\mathbf{0})$

$\begin{array}{lrrrrrr} & F=0 & F=20 & F=40 & F=60 & F=80 & F=100 \\ \text { bs-BLYP } & 0.9579 & 0.9602 & 0.9894 & 0.9989 & 0.9418 & 0.7959 \\ & -544.26082 & -544.25834 & -544.24663 & -544.23466 & -544.22769 & -544.21695 \\ \text { bs-B3LYP } & 0.9925 & 0.9909 & 1.0023 & 1.0004 & 0.942 & 0.7741 \\ & -544.33228 & -544.33637 & -544.32772 & -544.31669 & -544.30755 & -544.29013 \\ \text { bs-mPWPW91 } & 0.9667 & 0.9685 & 0.9925 & 0.9982 & 0.9432 & 0.8041 \\ & -544.51840 & -544.51833 & -544.50788 & -544.49671 & -544.49002 & -544.47890 \\ \text { bs-mPW1PW91 } & 0.999 & 0.9971 & 1.005 & 1.0018 & 0.9478 & 0.7868 \\ & -544.30153 & -544.30888 & -544.30164 & -544.29108 & -544.28141 & -544.26213 \\ \text { bs-MPW1K } & 1.0055 & 1.0018 & 1.0079 & 1.0099 & 0.9673 & 0.8023 \\ \text { bs-TPSS } & -544.15879 & -544.17102 & -544.16510 & -544.15344 & -544.14067 & -544.11628 \\ & 0.9831 & 0.9842 & 1.0001 & 0.9956 & 0.9345 & 0.7878 \\ \text { bs-TPSSh } & -544.21141 & -544.21094 & -544.20077 & -544.19078 & -544.18563 & -544.17636 \\ & 0.9954 & 0.9951 & 1.0045 & 0.9963 & 0.935 & 0.7795 \\ & -544.14400 & -544.14660 & -544.13784 & -544.12820 & -544.12181 & -544.10889\end{array}$


$\left\{\left(\mathrm{NH}_{3}\right) \mathrm{Cu}\right\}_{2} \mathrm{O}_{2}{ }^{2+}$ (1)

\begin{tabular}{lr} 
& \multicolumn{1}{c}{$F=0$} \\
bs-BLYP & 0.9499 \\
& -657.61694 \\
bs-B3LYP & 0.9902 \\
& -657.73269 \\
bs-mPWPW91 & 0.9592 \\
& -657.90501 \\
bs-mPW1PW91 & 0.9981 \\
& -657.65962 \\
bs-MPW1K & 1.0066 \\
& -657.49700 \\
bs-TPSS & 0.9764 \\
& -657.61863 \\
bs-TPSSh & 0.9918 \\
& -657.53406
\end{tabular}

$$
F=20
$$

0.9625

$-657.62094$

0.9972

$-657.73873$

0.9698

$-657.91030$

1.0031

$-657.66683$

1.0105

$-657.50493$

0.9849

$-657.62409$

0.9978

$-657.54033$
$F=40$

0.9951

$-657.61808$

1.0066

$-657.73769$

0.9970

$-657.90860$

1.0076

$-657.66693$

1.0124

$-657.50613$

1.0024

$-657.62271$

1.0057

$-657.53965$

$$
\begin{aligned}
& F=60 \\
& 0.9891
\end{aligned}
$$

$-657.61072$

0.9761

$-657.73148$

0.9889

$-657.90228$

0.9763

$-657.66175$

0.9824

$-657.50184$

0.9827

$-657.61703$

0.9760

$-657.53433$

$$
\begin{aligned}
& F=80 \\
& 0.8793
\end{aligned}
$$

$-657.60241$

0.8547

$-657.72309$

0.8860

$-657.89503$

0.8635

$-657.65416$

0.8745

$-657.49430$

0.8744

$-657.61085$

0.8632

$-657.52779$

$$
F=100
$$

0.6975

$-657.59309$

0.4645

$-657.71210$

0.7157

$-657.88713$

0.6700

$-657.64376$

0.6688

$-657.48294$

0.7016

$-657.60428$

0.6815

$-657.51987$
$\left\{\left(\mathrm{NH}_{3}\right)_{2} \mathrm{Cu}_{2} \mathrm{O}_{2}{ }^{2+}(\mathbf{2})\right.$

\begin{tabular}{lr} 
& \multicolumn{1}{c}{$F=0$} \\
bs-BLYP & 0.943 \\
bs-B3LYP & -770.88656 \\
& 0.967 \\
bs-mPWPW91 & 0.947 \\
& -771.21353 \\
bs-mPW1PW91 & 0.978 \\
& -770.95776
\end{tabular}

$$
F=20
$$

0.912

$-770.85389$

$$
0.932
$$

$-771.03214$

$$
0.918
$$

$-771.18730$

0.947

$-770.93173$

0.977

87459

1.020
$F=60$

0.514

$-770.77353$

0.801

$-770.95505$

0.549

$-771.11373$

0.619

$-770.86002$

0.653
$F=80$

0.000

$-770.80206$

0.578

$-770.98349$

0.046

$-771.04722$

0.059

$-770.88742$

0.872
$F=100$ 0.000

$-770.80915$ 0.602

$-771.01233$ 0.000

$-771.14249$

0.697

$-770.91940$

0.883 


$\begin{array}{lrrrrrr} & -770.78981 & -770.76281 & -770.70564 & -770.69262 & -770.72114 & -770.78030 \\ \text { bs-TPSS } & 0.949 & 0.917 & 0.943 & 0.553 & 0.000 & 0.000 \\ & -770.95120 & -770.92473 & -770.86729 & -770.85293 & -770.87478 & -770.89117 \\ \text { bs-TPSSh } & 0.960 & 0.927 & 0.999 & 0.602 & 0.055 & 0.386 \\ & -770.85591 & -770.82957 & -770.77222 & -770.75830 & -770.78799 & -770.80741\end{array}$

$\left\{\left(\mathrm{NH}_{3}\right)_{3} \mathrm{Cu}\right\}_{2} \mathrm{O}_{2}{ }^{2+}(\mathbf{3})$

$\begin{array}{lrrrrrr} & F=0 & F=20 & F=40 & F=60 & F=80 & F=100 \\ \text { bs-BLYP } & 0.982 & 0.992 & 0.997 & 0.785 & 0.233 & 0.000 \\ & -884.06285 & -884.04933 & -884.02597 & -884.01824 & -884.02362 & -883.96918 \\ \text { bs-B3LYP } & 0.962 & 0.985 & 0.990 & 0.775 & 0.249 & 0.687 \\ & -884.28884 & -884.28086 & -884.25986 & -884.25361 & -884.25745 & -884.26968 \\ \text { bs-mPWPW91 } & 0.983 & 0.994 & 0.998 & 0.811 & 0.295 & 0.000 \\ & -884.42599 & -884.41760 & -884.39758 & -884.39064 & -884.39434 & -884.33656 \\ \text { bs-mPW1PW91 } & 0.969 & 0.989 & 0.992 & 0.805 & 0.340 & 0.766 \\ \text { bs-MPW1K } & -884.14863 & -884.14541 & -884.12744 & -884.12232 & -884.12488 & -884.14028 \\ & 0.985 & 0.998 & 0.993 & 0.826 & 0.375 & 0.923 \\ \text { bs-TPSS } & -883.96831 & -883.96850 & -883.95261 & -883.94896 & -883.95109 & -883.99421 \\ & 0.978 & 0.992 & 0.998 & 0.815 & 0.310 & 0.029 \\ \text { bs-TPSSh } & -884.19339 & -884.18474 & -884.16485 & -884.15933 & -884.16515 & -884.11137 \\ & 0.968 & 0.988 & 0.995 & 0.881 & 0.319 & 0.511 \\ & -884.08223 & -884.07563 & -884.05637 & -884.05129 & -884.05633 & -884.05778\end{array}$




\section{Software}

All DFT calculations were carried out using Gaussian03 [1]. All CASSCF, CASPT2, and MS-CASPT2 calculations were carried out using MOLCAS6.2 [2]. All CC and $\mathrm{MBPT}(2)=\mathrm{MP} 2$ calculations performed in this work, except $\mathrm{CR}-\mathrm{CC}(2,3)$, were carried out with the routines from the Michigan State University package of CC computer codes [3] that form an integral part of the GAMESS package [4]. The CR-CC $(2,3)$ calculations were performed with the computer programs described in references [5] and [6] that form part of GAMESS as well.

[1] Frisch, M. J.; Trucks, G. W.; Schlegel, H. B.; Scuseria, G. E.; Robb, M. A.; Cheeseman, J. R.; Montgomery, J. A.; Vreven, T.; Kudin, K. N.; Burant, J. C.; Millam, J. M.; Iyengar, S. S.; Tomasi, J.; Barone, V.; Mennucci, B.; Cossi, M.; Scalmani, G.; Rega, N.; Petersson, G. A.; Nakatsuji, H.; Hada, M.; Ehara, M.; Toyota, K.; Fukuda, R.; Hasegawa, J.; Ishida, M.; Nakajima, T.; Honda, Y.; Kitao, O.; Nakai, H.; Klene, M.; Li, X.; Knox, J. E.; Hratchian, H. P.; Cross, J. B.; Adamo, C.; Jaramillo, J.; Gomperts, R.; Stratmann, R. E.; Yazyev, O.; Austin, A. J.; Cammi, R.; Pomelli, C.; Ochterski, J. W.; Ayala, P. Y.; Morokuma, K.; Voth, G. A.; Salvador, P.; Dannenberg, J. J.; Zakrzewski, V. G.; Dapprich, S.; Daniels, A. D.; Strain, M. C.; Farkas, O.; Malick, D. K.; Rabuck, A. D.; Raghavachari, K.; Foresman, J. B.; Ortiz, J. V.; Cui, Q.; Baboul, A. G.; Clifford, S.; Cioslowski, J.; Stefanov, B. B.; Liu, G.; Liashenko, A.; Piskorz, P.; Komaromi, I.; Martin, R. L.; Fox, D. J.; Keith, T.; Al-Laham, M. A.; Peng, C. Y.; Nanayakkara, A.; Challacombe, M.; Gill, P. M. W.; Johnson, B.; Chen, W.; Wong, M. W.; Gonzalez, C.; Pople, J. A. Gaussian 03 (Revision B.05); Gaussian, Inc.: Pittsburgh, PA, 2003. 
[2] Karlström, G.; Lindh, R.; Malmqvist, P.-Å.; Roos, B. O.; Ryde, U.; Veryazov, V.; Widmark, P. O.; Cossi, M.; Schimmelpfennig, B.; Neogrady, P.; Seijo, L. Comput. Matl. Sci. 2003, 28, 222.

[3] Piecuch, P.; Kucharski, S.A.; Kowalski, K.; Musiał, M. Comp. Phys. Commun. 2002, 149, 71.

[4] Schmidt, M. W.; Baldridge, K. K.; Boatz, J. A.; Elbert, S. T.; Gordon, M. S.; Jensen, J. H.; Koseki, S.; Matsunaga, N.; Nguyen, K. A.; Su, S.; Windus, T. L.; Dupuis, M.; Montgomery, J. A. J. Comp. Chem. 1993, 14, 1347.

[5] Piecuch, P.; Włoch, M. J. Chem. Phys. 2005, 123, 224105.

[6] Piecuch, P.; Wloch, M.; Gour, J. R.; Kinal, A. Chem. Phys. Lett. 2006, 418, 467. 